\title{
Fujita type theorems for a class of semilinear parabolic equations with a gradient term
}

\author{
Yuanyuan Nie, Mingjun Zhou, Qian Zhou*, Yang Na \\ School of Mathematics, Jilin University, Changchun 130012, China.
}

Communicated by K. Q. Lan

\begin{abstract}
This paper concerns the asymptotic behavior of solutions to the Neumann exterior problem of a class of semilinear parabolic equations with a gradient term. The blow-up theorem of Fujita type is established and the critical Fujita exponent is formulated by spacial dimension, the behavior of the coefficient of the gradient term at infinity and other exponents. (C)2017 All rights reserved.
\end{abstract}

Keywords: Critical Fujita exponent, gradient term.

2010 MSC: 35B33, 35K20, 35K58.

\section{Introduction}

The purpose of paper is to investigate the Fujita type theorems for the homogeneous Neumann exterior problem

$$
\begin{array}{lr}
|x|^{\lambda_{1}} \frac{\partial u}{\partial t}=\Delta u+b(|x|) x \cdot \nabla u+|x|^{\lambda_{2}} u^{p}, & x \in \mathbb{R}^{n} \backslash \bar{B}_{1}, \quad t>0, \\
\frac{\partial u}{\partial v}(x, t)=0, & x \in \partial B_{1}, \quad t>0, \\
u(x, 0)=u_{0}(x), & x \in \mathbb{R}^{n} \backslash \bar{B}_{1},
\end{array}
$$

where $p>1,0 \leqslant \lambda_{1} \leqslant \lambda_{2}<p \lambda_{1}+(p-1) n, 0 \leqslant b \in C^{1}([1,+\infty))$ satisfies

$$
\lim _{s \rightarrow+\infty} s^{2} b(s)=\kappa, \quad(0 \leqslant \kappa \leqslant+\infty),
$$

$0 \leqslant u_{0} \in C_{0}\left(\mathbb{R}^{n} \backslash B_{1}\right)$ satisfies $\left.\frac{\partial u_{0}}{\partial v}\right|_{\partial B_{1}}=0, B_{1}$ is the unit ball in $\mathbb{R}^{n}$ and $v$ denotes the unit inner normal vector to $\partial \mathrm{B}_{1}$.

The critical exponents for diffusion equations with nonlinear internal heat source has attached extensive attention since 1966, when Fujita [6] proved that for the Cauchy problem of the equation (1.1)

\footnotetext{
*Corresponding author

Email address: zhouqian@jlu.edu.cn (Qian Zhou)
}

doi:10.22436/jnsa.010.04.26 
with $\mathrm{b} \equiv 0$ and $\lambda_{1}=\lambda_{2}=0$, the nontrivial nonnegative solution blows up in a finite time if $1<\mathrm{p}<$ $p_{c}=1+2 / n$, whereas exists globally for small initial data and blows up in a finite time for large ones if $p>p_{c}=1+2 / n$. This result fully reveals that the exponent $p$ of the nonlinear reaction plays a remarkable role in affecting the properties of solutions. We call $p_{c}$ with the above properties the critical Fujita exponent and the similar result a blow-up theorem of Fujita type.

There have been many kinds of extensions of Fujita's results since then, such as different types of parabolic equations and systems with or without degeneracies or singularities, various geometries of domains, different nonlinear reactions or nonhomogeneous boundaries, etc. One can see the survey papers [3, 7] and the references therein, and more recent works $[2,4,9,11-18,20]$. For the semilinear parabolic equations, which are simple but not insignificant, Aguirre and Escobedo [1] showed that

$$
p_{c}=1+2 /(n+1)
$$

is the critical Fujita exponent of the Cauchy problem of

$$
\frac{\partial u}{\partial t}=\Delta u+b_{0} \cdot \nabla u+u^{p}, \quad x \in \mathbb{R}^{n}, t>0,
$$

with $\mathbf{b}_{0}$ be a nonzero constant vector. Then, Wang and Zheng [13] considered the Cauchy problem of (1.1) with $b \equiv 0$ and the critical Fujita exponent was formulated as

$$
p_{c}=1+\left(2+\lambda_{2}\right) /\left(n+\lambda_{1}\right) .
$$

Recently in [20], the Cauchy problem of (1.1) with $\lambda_{1}=\lambda_{2}=0$ was studied and it was shown that

$$
p_{c}= \begin{cases}1, & \kappa=+\infty, \\ 1+2 /(n+\kappa), & -n<k<+\infty, \\ +\infty, & -\infty \leqslant \kappa \leqslant-n .\end{cases}
$$

As to Neumann exterior problems, Levine and Zhang [8] investigated the critical Fujita exponent of the homogeneous Neumann exterior problem of (1.1) with $b \equiv 0$ and $\lambda_{1}=\lambda_{2}=0$, and proved that $p_{c}$ is still $1+2 / n$ which is the same as that Fujita proved. where $\Omega \subset \mathbb{R}^{n}$ with $n \geqslant 3$ is a domain with bounded complement and $v$ denotes the unit inner normal vector to $\partial \Omega$. In [19], the authors concerned the problem (1.1), (1.2), (1.3) with

$$
\mathrm{b}(\mathrm{s})=\frac{\kappa}{\mathrm{s}^{2}}, \quad \mathrm{~s}>0, \quad(-\infty<\kappa<+\infty)
$$

and formulated the critical Fujita exponent as

$$
p_{c}= \begin{cases}1+\left(2+\lambda_{2}\right) /\left(n+k+\lambda_{1}\right), & \kappa>-n-\lambda_{1}, \\ +\infty, & \kappa \leqslant-n-\lambda_{1} .\end{cases}
$$

In this paper, we investigate the Fujita type theorems for the Neumann exterior problem (1.1)-(1.3). It is proved that the critical Fujita exponent to the problem is

$$
p_{c}= \begin{cases}1, & k=+\infty, \\ 1+\left(2+\lambda_{2}\right) /\left(n+k+\lambda_{1}\right), & 0 \leqslant k<+\infty .\end{cases}
$$

That is to say, if $1<p<p_{c}$, there does not exist any nontrivial nonnegative global solution, whereas if $p>p_{c}$, there exist both nontrivial nonnegative global and blow-up solutions. The technique used in this paper is mainly inspired by $[10,13,19,20]$. To prove the blow-up of solutions, we use precise energy integral estimates instead of constructing subsolutions. For the global existence of nontrivial solutions, 
we construct a nontrivial global supersolution. It should be noted that we have to seek a complicated supersolution and do some precise calculations in order to overcome the difficulty from the non-selfsimilar construction of (1.1).

The paper is organized as follows. Some preliminaries and main results are listed in Section 2, such as the well-posedness of the problem (1.1)-(1.3) and some auxiliary lemmas to be used later, as well as the blow-up theorems of Fujita type. The proofs of the main results are obtained in Section 3.

\section{Preliminaries and main results}

The solutions to the problem (1.1)-(1.3) are defined as follows.

Definition 2.1. A nonnegative function $u$ is called a solution to the problem (1.1)-(1.3) in $(0, T)$ with $0<\mathrm{T} \leqslant+\infty$, if

$$
u \in C\left([0, T), \mathrm{L}_{\text {loc }}^{1}\left(\mathbb{R}^{n} \backslash \mathrm{B}_{1}\right)\right) \cap \mathrm{L}_{\text {loc }}^{\infty}\left(0, \mathrm{~T} ; \mathrm{L}^{\infty}\left(\mathbb{R}^{\mathrm{n}} \backslash \mathrm{B}_{1}\right)\right),
$$

and the integral identity

$$
\begin{aligned}
& \int_{0}^{T} \int_{\mathbb{R}^{n} \backslash B_{1}}|x|^{\lambda_{1}} u(x, t) \frac{\partial \varphi}{\partial t}(x, t) d x d t+\int_{0}^{T} \int_{\mathbb{R}^{n} \backslash B_{1}} u(x, t)(\Delta \varphi(x, t)-\operatorname{div}(b(|x|) \varphi(x, t) x)) d x d t \\
& \quad+\int_{0}^{T} \int_{\mathbb{R}^{\mathfrak{n}} \backslash B_{1}}|x|^{\lambda_{2}} u^{p}(x, t) \varphi(x, t) d x d t-\int_{0}^{T} \int_{\partial B_{1}} u(x, t)\left(\frac{\partial \varphi}{\partial v}(x, t)-|x|^{2} b(|x|) \varphi(x, t)\right) d \sigma d t \\
& \quad+\int_{\mathbb{R}^{n} \backslash B_{1}}|x|^{\lambda_{1}} u_{0}(x) \varphi(x, 0) d x=0,
\end{aligned}
$$

holds for any $0 \leqslant \varphi \in C^{2,1}\left(\left(\mathbb{R}^{n} \backslash B_{1}\right) \times[0, T)\right)$ vanishing when $t$ near $T$ or $|x|$ being sufficiently large.

Definition 2.2. A solution $u$ to the problem (1.1)-(1.3) is called a blow-up solution, if there exists some $\mathrm{T}_{*} \in(0,+\infty)$, which is called blow-up time, such that

$$
\|\mathrm{u}(\cdot, \mathrm{t})\|_{\mathrm{L}^{\infty}\left(\mathbb{R}^{n} \backslash B_{1}\right)} \rightarrow+\infty, \quad \text { as } \mathrm{t} \rightarrow \mathrm{T}_{*}^{-} .
$$

Otherwise, $u$ is called a global solution.

For $0 \leqslant u_{0} \in C_{0}\left(\mathbb{R}^{n} \backslash B_{1}\right)$ with $\left.\frac{\partial u_{0}}{\partial v}\right|_{\partial B_{1}}=0,0 \leqslant b \in C^{1}([1,+\infty))$ and $p>1$, one can establish the existence, uniqueness and the comparison principle to the local in time solutions of (1.1)-(1.3) by use of the classical theory on parabolic equations (see, e.g., [5]).

The Fujita type theorems for the problem (1.1)-(1.3) are stated as follows.

Theorem 2.3. Assume that $0 \leqslant \mathrm{~b} \in \mathrm{C}^{1}([1,+\infty))$ satisfies (1.4) with $0 \leqslant \mathrm{k}<+\infty$. Let $1<\mathrm{p}<\mathrm{p}_{\mathrm{c}}$. Then for any nontrivial $0 \leqslant \mathrm{u}_{0} \in \mathrm{C}_{0}\left(\mathbb{R}^{\mathrm{n}} \backslash \mathrm{B}_{1}\right),\left.\frac{\partial \mathrm{u}_{0}}{\partial v}\right|_{\partial \mathrm{B}_{1}}=0$, the solution to the problem (1.1)-(1.3) must blow up in a finite time.

Theorem 2.4. Assume that $0 \leqslant \mathrm{~b} \in \mathrm{C}^{1}([1,+\infty))$ satisfies (1.4) with $0 \leqslant \mathrm{k} \leqslant+\infty$. Let $\mathrm{p}>\mathrm{p}_{\mathrm{c}}$. Then there exist both nontrivial nonnegative global and blow-up solutions to the problem (1.1)-(1.3).

\section{Proofs of main results}

To prove Theorem 2.3, the following auxiliary lemma is necessary. We omit the proof in consideration of the similar method in $[19,20]$. 
Lemma 3.1. Assume that $0 \leqslant \mathrm{~b} \in \mathrm{C}^{1}([1,+\infty))$ satisfies (1.4) with $0 \leqslant \mathrm{k}<+\infty$, $\mathrm{u}$ be a solution to the problem (1.1)-(1.3), and

$$
\psi_{R}(r)= \begin{cases}h(r), & 1 \leqslant r \leqslant R \\ \frac{1}{2} h(r)\left(1+\cos \frac{(r-R) \pi}{(\delta-1) R}\right), & R<r<\delta R \\ 0, & r \geqslant \delta R\end{cases}
$$

with

$$
h(r)=\exp \left\{\int_{1}^{r} s b(s) d s\right\}, \quad r \geqslant 1
$$

Then there exist three numbers $R_{0}>1, \delta>1$ and $M_{0}>0$ depending only on $n$ and $b$, such that for any $R>R_{0}$,

$$
\begin{aligned}
\frac{d}{d t} \int_{\mathbb{R}^{n} \backslash B_{1}}|x|^{\lambda_{1}} u(x, t) \psi_{R}(|x|) d x \geqslant & -M_{0} R^{-2} \int_{B_{\delta R} \backslash B_{R}} u(x, t) \psi_{R}(|x|) d x \\
& +\int_{\mathbb{R}^{n} \backslash B_{1}}|x|^{\lambda_{2}} u^{p}(x, t) \psi_{R}(|x|) d x, \quad t>0,
\end{aligned}
$$

in the distribution sense, where $B_{r}$ denotes the open ball in $\mathbb{R}^{n}$ with radius $r$ and centered at the origin.

Remark 3.2. For the case $k=+\infty$, one can prove that (3.1) holds for each fixed $R>1$, but $\delta>1$ and $\mathrm{M}_{0}>0$ depend also on $\mathrm{R}$.

Proof of Theorem 2.3. We claim that $\psi_{R}, h, R_{0}, \delta$ and $M_{0}$ are introduced in Lemma 3.1. It follows from $0 \leqslant k<+\infty$ and $1<p<p_{c}$ that

$$
\kappa<\frac{2+\lambda_{2}}{p-1}-n-\lambda_{1}
$$

Fix $\tilde{\kappa}$ to satisfy

$$
\kappa<\tilde{\kappa}<\frac{2+\lambda_{2}}{p-1}-\mathrm{n}-\lambda_{1}
$$

which together with (1.4), yields that there exists $R_{1}>1$ such that

$$
0 \leqslant s^{2} b(s)<\tilde{\kappa}, \quad s>R_{1} .
$$

For any $R>R_{1}$, one can get that

$$
\begin{aligned}
0 \leqslant \psi_{R}(|x|) & \leqslant h(|x|) \chi_{[1, \delta R]}(|x|) \\
& \leqslant \exp \left\{\int_{0}^{\delta R} \operatorname{sb}(s) d s\right\} \chi_{[1, \delta R]}(|x|) \\
& \leqslant \exp \left\{\int_{0}^{R_{1}} \operatorname{sb}(s) d s\right\} \exp \left\{\tilde{\kappa} \int_{R_{1}}^{\delta R} \frac{1}{s} d s\right\} \chi_{[1, \delta R]}(|x|) \\
& =M^{\tilde{\kappa}^{\tilde{\kappa}}} \chi_{[1, \delta R]}(|x|), \quad|x| \geqslant 1,
\end{aligned}
$$

where $\chi_{[1, \delta R]}$ is the characteristic function of the interval $[1, \delta R]$, while $M>0$ depends only on $n, b, R_{1}, \delta$ and $\tilde{\kappa}$. Let $u$ be the solution to the problem (1.1)-(1.3), and denote

$$
w_{R}(t)=\int_{\mathbb{R}^{n} \backslash B_{1}}|x|^{\lambda_{1}} u(x, t) \psi_{R}(x) d x, \quad t \geqslant 0 .
$$


For any $R>\max \left\{R_{0}, R_{1}\right\}$, Lemma 3.1 implies

$$
\frac{d}{d t} w_{R}(t) \geqslant-M_{0} R^{-2} \int_{B_{\delta R} \backslash B_{R}} u(x, t) \psi_{R}(|x|) d x+\int_{\mathbb{R}^{n} \backslash B_{1}}|x|^{\lambda_{2}} u^{p}(x, t) \psi_{R}(|x|) d x, \quad t>0 .
$$

The Hölder inequality and (3.3) yield

$$
\begin{array}{rl}
\int_{B_{\delta R} \backslash B_{R}} & u(x, t) \psi_{R}(|x|) d x \\
\leqslant & \left(\int_{B_{\delta R} \backslash B_{R}}|x|^{-\lambda_{2} /(p-1)} \psi_{R}(|x|) d x\right)^{(p-1) / p}\left(\int_{B_{\delta R} \backslash B_{R}}|x|^{\lambda_{2}} u^{p}(x, t) \psi_{R}(|x|) d x\right)^{1 / p} \\
\leqslant & \left(M R^{\tilde{\kappa}-\lambda_{2} /(p-1)} \int_{B_{\delta R} \backslash B_{R}} d x\right)^{(p-1) / p}\left(\int_{B_{\delta R} \backslash B_{R}}|x|^{\lambda_{2}} u^{p}(x, t) \psi_{R}(|x|) d x\right)^{1 / p} \\
\leqslant & M_{1}^{(p-1) / p} R^{n+\tilde{\kappa}-\left(n+\tilde{\kappa}+\lambda_{2}\right) / p}\left(\int_{\mathbb{R}^{n} \backslash B_{1}}|x|^{\lambda_{2}} u^{p}(x, t) \psi_{R}(|x|) d x\right)^{1 / p}, \quad t>0,
\end{array}
$$

with $M_{1}=M\left(\delta^{n}-1\right) \omega_{n}>0$ and $\omega_{n}$ is the volume of the unit ball in $\mathbb{R}^{n}$. Substituting (3.5) into (3.4) gives

$$
\begin{aligned}
& \frac{d}{d t} \mathcal{w}_{R}(t) \geqslant-M_{0} M_{1}^{(p-1) / p} R^{n+\tilde{\kappa}-2-\left(n+\tilde{\kappa}+\lambda_{2}\right) / p}\left(\int_{\mathbb{R}^{n} \backslash B_{1}}|x|^{\lambda_{2}} u^{p}(x, t) \psi_{R}(|x|) d x\right)^{1 / p} \\
& +\int_{\mathbb{R}^{n} \backslash B_{1}}|x|^{\lambda_{2}} u^{p}(x, t) \psi_{R}(|x|) d x, \quad t>0 .
\end{aligned}
$$

It follows from $\lambda_{2}<p \lambda_{1}+(p-1) n$ that $n+\left(p \lambda_{1}-\lambda_{2}\right) /(p-1)>0$, which together with the Hölder inequality and (3.3), leads to

$$
\begin{array}{rl}
\int_{\mathbb{R}^{n} \backslash B_{1}}|x|^{\lambda_{1}} & u(x, t) \psi_{R}(|x|) d x \\
& \leqslant\left(\int_{\mathbb{R}^{\mathfrak{n}} \backslash B_{1}}|x|^{\left(p \lambda_{1}-\lambda_{2}\right) /(p-1)} \psi_{R}(|x|) d x\right)^{(p-1) / p}\left(\int_{\mathbb{R}^{n} \backslash B_{1}}|x|^{\lambda_{2}} u^{p}(x, t) \psi_{R}(|x|) d x\right)^{1 / p} \\
& \leqslant\left(M R^{\tilde{\kappa}} \int_{B_{\delta R} \backslash B_{1}} r^{\left(p \lambda_{1}-\lambda_{2}\right) /(p-1)} d x\right)^{(p-1) / p}\left(\int_{\mathbb{R}^{n} \backslash B_{1}}|x|^{\lambda_{2}} u^{p}(x, t) \psi_{R}(|x|) d x\right)^{1 / p} \\
& =\left(M R^{\tilde{\kappa}} n \omega_{n} \int_{1}^{\delta R} r^{n-1+\left(p \lambda_{1}-\lambda_{2}\right) /(p-1)} d r\right)^{(p-1) / p}\left(\int_{\mathbb{R}^{n} \backslash B_{1}}|x|^{\lambda_{2}} u^{p}(x, t) \psi_{R}(|x|) d x\right)^{1 / p} \\
& \leqslant M_{2}^{(p-1) / p} R^{n+\tilde{\kappa}+\lambda_{1}-\left(n+\tilde{\kappa}+\lambda_{2}\right) / p}\left(\int_{\mathbb{R}^{\mathfrak{n}} \backslash B_{1}}|x|^{\lambda_{2}} u^{p}(x, t) \psi_{R}(|x|) d x\right)^{1 / p}, \quad t>0,
\end{array}
$$

with $M_{2}=\frac{M n(p-1) \omega_{n} \delta^{n}}{n(p-1)+p \lambda_{1}-\lambda_{2}}>0$, then

$$
\int_{\mathbb{R}^{n} \backslash B_{1}}|x|^{\lambda_{2}} u^{p}(x, t) \psi_{R}(|x|) d x \geqslant M_{2}^{-(p-1)} R^{-(p-1)\left(n+\tilde{\kappa}+\lambda_{1}\right)+\lambda_{1}-\lambda_{2}} w_{R}^{p}(t), \quad t>0 .
$$

Substituting (3.7) into (3.6), one gets that for any $R>\max \left\{R_{0}, R_{1}\right\}$,

$$
\begin{aligned}
\frac{\mathrm{d}}{\mathrm{dt}} w_{R}(\mathrm{t}) & \geqslant-M_{0}\left(\frac{M_{1}}{M_{2}}\right)^{(\mathrm{p}-1) / p} \mathrm{R}^{-2-\lambda_{1}} w_{R}(\mathrm{t})+M_{2}^{-(p-1)} R^{-(p-1)\left(n+\tilde{\kappa}+\lambda_{1}\right)+\lambda_{1}-\lambda_{2}} w_{R}^{p}(t) \\
& =w_{R}(t)\left(-M_{0}\left(\frac{M_{1}}{M_{2}}\right)^{(p-1) / p} R^{-2-\lambda_{1}}+M_{2}^{-(p-1)} R^{-(p-1)\left(n+\tilde{\kappa}+\lambda_{1}\right)+\lambda_{1}-\lambda_{2}} w_{R}^{p-1}(t)\right), \quad t>0 .
\end{aligned}
$$


Notice that (3.2) implies

$$
-2-\lambda_{1}<-(p-1)\left(n+\tilde{\kappa}+\lambda_{1}\right)+\lambda_{1}-\lambda_{2},
$$

while $w_{R}(0)$ is nondecreasing with respect to $R \in(1,+\infty)$ and

$$
\sup \left\{w_{R}(0): R>1\right\}>0 .
$$

Therefore, there exists $R_{2}>1$ such that for any $R>R_{2}$,

$$
M_{0}\left(\frac{M_{1}}{M_{2}}\right)^{(p-1) / p} R^{-2-\lambda_{1}} \leqslant \frac{1}{2} M_{2}^{-(p-1)} R^{-(p-1)\left(n+\tilde{\kappa}+\lambda_{1}\right)+\lambda_{1}-\lambda_{2}} w_{R}^{p-1}(0) .
$$

Fix $R>\max \left\{R_{0}, R_{1}, R_{2}\right\}$. Then, (3.8) and (3.9) yield that

$$
\frac{d}{d t} w_{R}(t) \geqslant \frac{1}{2} M_{2}^{-(p-1)} R^{-(p-1)\left(n+\tilde{\kappa}+\lambda_{1}\right)+\lambda_{1}-\lambda_{2}} w_{R}^{p}(t), \quad t>0 .
$$

Since $p>1$, there exists $T_{*}>0$ such that

$$
\mathcal{w}_{\mathrm{R}}(\mathrm{t})=\int_{\mathbb{R}^{\mathfrak{n}} \backslash \mathrm{B}_{1}}|x|^{\lambda_{1}} \mathfrak{u}(x, \mathrm{t}) \psi_{\mathrm{R}}(|x|) \mathrm{d} x \rightarrow+\infty, \quad \text { as } \quad \mathrm{t} \rightarrow \mathrm{T}_{*}^{-} .
$$

It follows from supp $\psi_{R}(|x|)=\bar{B}_{\delta R} \backslash B_{1}$ that

$$
\|\mathrm{u}(\cdot, \mathrm{t})\|_{\mathrm{L}^{\infty}\left(\mathbb{R}^{\mathfrak{n}} \backslash \mathrm{B}_{1}\right)} \rightarrow+\infty, \quad \text { as } \mathrm{t} \rightarrow \mathrm{T}_{*}^{-},
$$

i.e., $u$ blows up in a finite time.

Now, let us prove Theorem 2.4. Firstly, we study self-similar supersolutions of (1.1) of the form

$$
u(x, t)=(t+\tau)^{-\alpha} v\left((t+\tau)^{-\beta}|x|\right), \quad x \in \mathbb{R}^{n} \backslash B_{1}, \quad t \geqslant 0,
$$

with

$$
\alpha=\frac{2+\lambda_{2}}{\left(2+\lambda_{1}\right)(p-1)}, \quad \beta=\frac{1}{2+\lambda_{1}}
$$

and $\tau>0$ will be determined. If $v \in C^{1,1}([0,+\infty))$ solves

$$
v^{\prime \prime}(r)+\frac{n-1}{r} v^{\prime}(r)+(t+\tau)^{2 \beta} r b\left((t+\tau)^{\beta} r\right) v^{\prime}(r)+\beta r^{1+\lambda_{1}} v^{\prime}(r)+\alpha r^{\lambda_{1}} v(r)+r^{\lambda_{2}} v^{p}(r) \leqslant 0, \quad r>0,
$$

then $u$ given by (3.10) is a supersolution to (1.1). Moreover, $v^{\prime}(r) \leqslant 0(r>0)$ implies $\left.\frac{\partial u}{\partial v}\right|_{\partial B_{1} \times(0,+\infty)} \geqslant 0$.

Lemma 3.3. Assume that $0 \leqslant \mathrm{~b} \in \mathrm{C}^{1}([1,+\infty))$ satisfies (1.4) with $0 \leqslant \mathrm{k} \leqslant+\infty, \mathrm{p}>\mathrm{p}_{\mathrm{c}}$,

$$
v(r)=\eta e^{-A(r)}, \quad r \geqslant 0,
$$

with $\mathrm{A} \in \mathrm{C}^{1,1}([0,+\infty))$ satisfies $\mathrm{A}(0)=0$ and

$$
A^{\prime}(r)= \begin{cases}A_{1} r^{1+\lambda_{1}}, & 0 \leqslant r \leqslant l^{2}, \\ A_{2} r^{1+\lambda_{1}}+\left(A_{1}-A_{2}\right) \frac{l^{2\left(n+\kappa_{2}+\lambda_{1}\right)}}{r^{n+\kappa_{2}-1}}, & l^{2}<r<l, \\ A_{2} r^{1+\lambda_{1}}+\left(A_{1}-A_{2}\right) l^{n+\kappa_{2}+\lambda_{1}} r^{1+\lambda_{1}}, & r \geqslant l,\end{cases}
$$


where $0<\mathrm{l}<1$ to be determined,

$$
A_{i}=\frac{2\left(2+\lambda_{2}\right)}{\left(2+\lambda_{1}\right)\left(n+\kappa_{i}+\lambda_{1}\right)\left(p+p_{c}-2\right)}, \quad i=1,2,
$$

with $-\mathrm{n}<\mathrm{\kappa}_{1}<\mathrm{\kappa}_{2}<\mathrm{\kappa}$ satisfying

$$
\inf \left\{s^{2} b(s): s>0\right\}>\kappa_{1}, \quad\left(n+\kappa_{2}+\lambda_{1}\right)\left(p+p_{c}-2\right)>2\left(2+\lambda_{2}\right) .
$$

Then, there exist $0<l<1, \tau>0$ and $\eta>0$ such that $u$ given by (3.10) and (3.11) is a supersolution to (1.1). Furthermore,

$$
\left.\frac{\partial u}{\partial v}\right|_{\partial B_{1} \times(0,+\infty)} \geqslant 0
$$

Proof. Denote $\kappa_{0}=\inf \left\{s^{2} b(s): s>0\right\}$. For $0<r<l^{2}$,

$$
\begin{aligned}
v^{\prime \prime}(r) & +\frac{n-1}{r} v^{\prime}(r)+(t+\tau)^{2 \beta} r b\left((t+\tau)^{\beta} r\right) v^{\prime}(r)+\beta r^{1+\lambda_{1}} v^{\prime}(r)+\alpha r^{\lambda_{1}} v(r) \\
= & r^{\lambda_{1}} v(r)\left(-A_{1}\left(1+\lambda_{1}\right)+A_{1}^{2} r^{2+\lambda_{1}}-(n-1) A_{1}-A_{1}(t+\tau)^{2 \beta} r^{2} b\left((t+\tau)^{\beta} r\right)-A_{1} \beta r^{2+\lambda_{1}}+\alpha\right) \\
\leqslant & r^{\lambda_{1}} v(r)\left(-\left(n+\kappa_{0}+\lambda_{1}\right) A_{1}+\frac{2+\lambda_{2}}{\left(2+\lambda_{1}\right)(p-1)}+\left(A_{1}-\beta\right) A_{1} r^{2+\lambda_{1}}\right) \\
& =\left(-\frac{2\left(2+\lambda_{2}\right)\left(\kappa_{0}-\kappa_{1}\right)}{\left(2+\lambda_{1}\right)\left(n+\kappa_{1}+\lambda_{1}\right)\left(p+p_{c}-2\right)}-\frac{\left(2+\lambda_{2}\right)\left(p-p_{c}\right)}{\left(2+\lambda_{1}\right)\left(p+p_{c}-2\right)(p-1)}+\left(A_{1}-\beta\right) A_{1} r^{2+\lambda_{1}}\right) r^{\lambda_{1}} v(r),
\end{aligned}
$$

which yields that there exists $0<l_{1}<1$ such that for any $0<l<l_{1}$,

$$
\begin{array}{r}
v^{\prime \prime}(r)+\frac{n-1}{r} v^{\prime}(r)+(t+\tau)^{2 \beta} b\left((t+\tau)^{\beta} r\right) r v^{\prime}(r)+\beta r^{1+\lambda_{1}} v^{\prime}(r)+\alpha r^{\lambda_{1}} v(r) \\
\leqslant-\frac{\left(2+\lambda_{2}\right)\left(p-p_{c}\right)}{2\left(2+\lambda_{1}\right)\left(p+p_{c}-2\right)(p-1)} r^{\lambda_{1}} v(r), \quad 0<r<l^{2}, \quad t>0 .
\end{array}
$$

For $\mathrm{l}^{2}<\mathrm{r}<\mathrm{l}$, it holds that

$$
\begin{aligned}
v^{\prime \prime}(r)+\frac{n+\kappa_{2}-1}{r} & v^{\prime}(r)+\beta r^{1+\lambda_{1}} v^{\prime}(r)+\alpha r^{\lambda_{1}} v(r) \\
= & v(r)\left(\left(A^{\prime}(r)\right)^{2}-A^{\prime \prime}(r)-\frac{n+\kappa_{2}-1}{r} A^{\prime}(r)-\beta r^{1+\lambda_{1}} A^{\prime}(r)+\alpha r^{\lambda_{1}}\right) \\
= & r^{\lambda_{1}} v(r)\left(\left(A_{2}+\left(A_{1}-A_{2}\right) \frac{l^{2\left(n+\kappa_{2}+\lambda_{1}\right)}}{r^{n+\kappa_{2}+\lambda_{1}}}\right)\left(A_{2}+\left(A_{1}-A_{2}\right) \frac{l^{2\left(n+\kappa_{2}+\lambda_{1}\right)}}{r^{n+\kappa_{2}+\lambda_{1}}}-\beta\right) r^{2+\lambda_{1}}\right. \\
& \left.\quad-\left(n+\kappa_{2}+\lambda_{1}\right) A_{2}+\frac{2+\lambda_{2}}{\left(2+\lambda_{1}\right)(p-1)}\right) \\
\leqslant & r^{\lambda_{1}} v(r)\left(-\frac{\left(2+\lambda_{2}\right)\left(p-p_{c}\right)}{\left(2+\lambda_{1}\right)\left(p+p_{c}-2\right)(p-1)}+\left(A_{1}-\beta\right) A_{1} r^{2+\lambda_{1}}\right),
\end{aligned}
$$

which implies that there exists $0<l_{2}<l_{1}$ such that for any $0<l<l_{2}$,

$$
v^{\prime \prime}(r)+\frac{n+\kappa_{2}-1}{r} v^{\prime}(r)+\beta r^{1+\lambda_{1}} v^{\prime}(r)+\alpha r^{\lambda_{1}} v(r) \leqslant-\frac{\left(2+\lambda_{2}\right)\left(p-p_{c}\right)}{2\left(2+\lambda_{1}\right)\left(p+p_{c}-2\right)(p-1)} r^{\lambda_{1}} v(r), \quad l^{2}<r<l .
$$

For $r>l$, one gets that

$$
v^{\prime \prime}(r)+\frac{n+\kappa_{2}-1}{r} v^{\prime}(r)+\beta r^{1+\lambda_{1}} v^{\prime}(r)+\alpha r^{\lambda_{1}} v(r)
$$




$$
\begin{aligned}
& =v(r)\left(\left(A_{2}+\left(A_{1}-A_{2}\right) l^{n+\kappa_{2}+\lambda_{1}}\right)^{2} r^{2+2 \lambda_{1}}-\left(n+\kappa_{2}+\lambda_{1}\right)\left(A_{2}+\left(A_{1}-A_{2}\right) l^{n+\kappa_{2}+\lambda_{1}}\right) r^{\lambda_{1}}\right. \\
& \left.\quad-\beta\left(A_{2}+\left(A_{1}-A_{2}\right) l^{n+\kappa_{2}+\lambda_{1}}\right) r^{2+2 \lambda_{1}}+\frac{2+\lambda_{2}}{\left(2+\lambda_{1}\right)(p-1)} r^{\lambda_{1}}\right) \\
& =r^{\lambda_{1}} v(r)\left(-\frac{\left(2+\lambda_{2}\right)\left(p-p_{c}\right)}{\left(2+\lambda_{1}\right)\left(p+p_{c}-2\right)(p-1)}-\left(n+\kappa_{2}+\lambda_{1}\right)\left(A_{1}-A_{2}\right) l^{n+\kappa_{2}+\lambda_{1}}\right) \\
& \quad+\left(A_{2}+\left(A_{1}-A_{2}\right) l^{n+\kappa_{2}+\lambda_{1}}\right)\left(A_{2}+\left(A_{1}-A_{2}\right) l^{n+\kappa_{2}+\lambda_{1}}-\beta\right) r^{2+2 \lambda_{1}} v(r) \\
& \leqslant-\frac{\left(2+\lambda_{2}\right)\left(p-p_{c}\right)}{2\left(2+\lambda_{1}\right)\left(p+p_{c}-2\right)(p-1)} r^{\lambda_{1}} v(r) \\
& \quad+\left(A_{2}+\left(A_{1}-A_{2}\right) l^{n+\kappa_{2}+\lambda_{1}}\right)\left(A_{2}+\left(A_{1}-A_{2}\right) l^{n+\kappa_{2}+\lambda_{1}}-\beta\right) r^{2+2 \lambda_{1}} v(r) .
\end{aligned}
$$

The choice of $\kappa_{1}, \kappa_{2}$ leads to

$$
\lim _{l \rightarrow 0^{+}}\left(A_{2}+\left(A_{1}-A_{2}\right) l^{n+\kappa_{2}+\lambda_{1}}\right)=A_{2}<\frac{1}{2+\lambda_{1}}=\beta,
$$

which yields that there exists $0<l_{3}<l_{2}$ such that for any $0<l<l_{3}$,

$$
A_{2}+\left(A_{1}-A_{2}\right) l^{n+\kappa_{2}+\lambda_{1}}<\beta,
$$

and thus

$$
v^{\prime \prime}(r)+\frac{n+\kappa_{2}-1}{r} v^{\prime}(r)+\beta r^{1+\lambda_{1}} v^{\prime}(r)+\alpha r^{\lambda_{1}} v(r) \leqslant-\frac{\left(2+\lambda_{2}\right)\left(p-p_{c}\right)}{2\left(2+\lambda_{1}\right)\left(p+p_{c}-2\right)(p-1)} r^{\lambda_{1}} v(r), \quad r>l .
$$

Fix $0<\mathrm{l}<\mathrm{l}_{3},(1.4)$ insures that one can choose $\tau>0$ sufficiently large such that

$$
(t+\tau)^{2 \beta} r b\left((t+\tau)^{\beta} r\right) \geqslant \frac{k_{2}}{r}, \quad r>l^{2}, \quad t>0 .
$$

It follows from (3.12), (3.13), (3.14), (3.15) that

$$
\begin{aligned}
& v^{\prime \prime}(r)+\frac{n-1}{r} v^{\prime}(r)+(t+\tau)^{2 \beta} r b\left((t+\tau)^{\beta} r\right) v^{\prime}(r)+\beta r^{1+\lambda_{1}} v^{\prime}(r)+\alpha r^{\lambda_{1}} v(r) \\
& \leqslant v^{\prime \prime}(r)+\frac{n+\kappa_{2}-1}{r} v^{\prime}(r)+\beta r^{1+\lambda_{1}} v^{\prime}(r)+\alpha r^{\lambda_{1}} v(r) \\
& \leqslant-\frac{\left(2+\lambda_{2}\right)\left(p-p_{c}\right)}{2\left(2+\lambda_{1}\right)\left(p+p_{c}-2\right)(p-1)} r^{\lambda_{1}} v(r), \quad r \in\left(0, l^{2}\right) \cup\left(l^{2}, l\right) \cup(l,+\infty), \quad t>0 .
\end{aligned}
$$

Due to $0 \leqslant \lambda_{1} \leqslant \lambda_{2}, p>1$ and the definition of $A$,

$$
0<M_{0}=\sup _{r>0} r^{\lambda_{2}-\lambda_{1}} e^{-(p-1) A(r)}<+\infty .
$$

Choose $\eta>0$ sufficiently small such that

$$
\eta^{p-1} \leqslant \frac{\left(2+\lambda_{2}\right)\left(p-p_{c}\right)}{2 M_{0}\left(2+\lambda_{1}\right)\left(p+p_{c}-2\right)(p-1)} .
$$

Then, (3.16) implies that

$$
\begin{aligned}
& v^{\prime \prime}(r)+\frac{n-1}{r} v^{\prime}(r)+(t+\tau)^{2 \beta} r b\left((t+\tau)^{\beta} r\right) v^{\prime}(r)+\beta r^{1+\lambda_{1}} v^{\prime}(r)+\alpha r^{\lambda_{1}} v(r)+r^{\lambda_{2}} v^{p}(r) \\
& \leqslant r^{\lambda_{1}} v(r)\left(-\frac{\left(2+\lambda_{2}\right)\left(p-p_{c}\right)}{2\left(2+\lambda_{1}\right)\left(p+p_{c}-2\right)(p-1)}+\eta^{p-1} r^{\lambda_{2}-\lambda_{1}} e^{-(p-1) A(r)}\right) \\
& \leqslant r^{\lambda_{1}} v(r)\left(-\frac{\left(2+\lambda_{2}\right)\left(p-p_{c}\right)}{2\left(2+\lambda_{1}\right)\left(p+p_{c}-2\right)(p-1)}+\eta^{p-1} M_{0}\right) \\
& \leqslant 0, \quad r \in\left(0, l^{2}\right) \cup\left(l^{2}, l\right) \cup(l,+\infty), \quad t>0 .
\end{aligned}
$$

Since $v \in C^{1,1}([0,+\infty))$, $u$ given by (3.10) and (3.11) is a supersolution to (1.1). 
Proof of Theorem 2.4. The comparison principle and Lemma 3.3 yield that there exists a nontrivial global solutions to the problem (1.1)-(1.3). We will show that the problem also admits a blow-up solutions. Fix $R>R_{0}$. Assume that $u$ is a solution to the problem (1.1)-(1.3). Lemma 3.1 and Remark 3.2 imply that

$$
\frac{d}{d t} w_{R}(t) \geqslant-M_{0} R^{-2} w_{R}(t)+\int_{\mathbb{R}^{n} \backslash B_{1}}|x|^{\lambda_{2}} u^{p}(x, t) \psi_{R}(|x|) d x, \quad t>0,
$$

where $\psi_{R}, R_{0}, \delta, M_{0}$ and $w_{R}(t)$ are given in Lemma 3.1, Remark 3.2 and the proof of Theorem 2.3. The Hölder inequality yields

$$
\begin{aligned}
& \int_{\mathbb{R}^{n} \backslash B_{1}}|x|^{\lambda_{1}} \mathfrak{u}(x, t) \psi_{R}(|x|) d x \\
& \leqslant\left(\int_{\mathbb{R}^{\mathfrak{n}} \backslash B_{1}}|x|^{\left(p \lambda_{1}-\lambda_{2}\right) /(p-1)} \psi_{R}(|x|) d x\right)^{(p-1) / p}\left(\int_{\mathbb{R}^{\mathfrak{n}} \backslash B_{1}}|x|^{\lambda_{2}} u^{p}(x, t) \psi_{R}(|x|) d x\right)^{1 / p} \\
& \leqslant\left(\int_{\mathbb{R}^{\mathfrak{n}} \backslash B_{1}}|x|^{p \lambda_{1} /(p-1)} \psi_{R}(|x|) d x\right)^{(p-1) / p}\left(\int_{\mathbb{R}^{\mathfrak{n}} \backslash B_{1}}|x|^{\lambda_{2}} u^{p}(x, t) \psi_{R}(|x|) d x\right)^{1 / p}, \quad t>0,
\end{aligned}
$$

which implies

$$
\int_{\mathbb{R}^{\mathfrak{n}} \backslash B_{1}}|x|^{\lambda_{2}} u^{p}(x, t) \psi_{R}(|x|) d x \geqslant\left(\int_{\mathbb{R}^{\mathfrak{n}} \backslash B_{1}}|x|^{p \lambda_{1} /(p-1)} \psi_{R}(|x|) d x\right)^{1-p} w_{R}^{p}(t), \quad t>0 .
$$

Substituting (3.18) into (3.17) to get

$$
\frac{d}{d t} w_{R}(t) \geqslant w_{R}(t)\left\{-M_{0} R^{-2}+\left(\int_{\mathbb{R}^{n} \backslash B_{1}}|x|^{p \lambda_{1} /(p-1)} \psi_{R}(|x|) d x\right)^{1-p} w_{R}^{p-1}(t)\right\}, \quad t>0 .
$$

If $\mathfrak{u}_{0}$ is so large that

$$
w_{R}(0)=\int_{\mathbb{R}^{\mathfrak{n}} \backslash B_{1}}|x|^{\lambda_{1}} u_{0}(x) \psi_{R}(|x|) d x \geqslant\left(2 M_{0} R^{-2}\right)^{1 /(p-1)} \int_{\mathbb{R}^{n} \backslash B_{1}}|x|^{p \lambda_{1} /(p-1)} \psi_{R}(|x|) d x,
$$

then (3.19) leads to

$$
\frac{\mathrm{d}}{\mathrm{dt}} w_{\mathrm{R}}(\mathrm{t}) \geqslant \frac{1}{2}\left(\int_{\mathbb{R}^{\mathrm{n}} \backslash \mathrm{B}_{1}}|x|^{\mathrm{p} \lambda_{1} /(p-1)} \psi_{\mathrm{R}}(|x|) \mathrm{d} x\right)^{1-\mathrm{p}} w_{\mathrm{R}}^{\mathrm{p}}(\mathrm{t}), \quad \mathrm{t}>0 .
$$

The same argument as in the proof of Theorem 2.3 shows that $u$ must blow up in a finite time.

Remark 3.4. For the critical case $p=p_{c}$ with $0 \leqslant \kappa<+\infty$, we need an additional condition (see [20]) that

$$
-\infty \leqslant \int_{1}^{+\infty} \frac{s^{2} \mathrm{~b}(\mathrm{~s})-\mathrm{k}}{\mathrm{s}} \mathrm{ds}<+\infty, \quad \text { if } \quad 0 \leqslant \kappa<+\infty .
$$

Similar to the proof in critical case in $[19,20]$, one can show the blow-up of solutions to the problem (1.1)-(1.3) for the critical case $p=p_{c}$ with $0 \leqslant \kappa<+\infty$, if (3.20) holds.

\section{Acknowledgment}

This work is supported by the National Natural Science Foundation of China (No. 11601182).

\section{References}

[1] J. Aguirre, M. Escobedo, On the blow-up of solutions of a convective reaction diffusion equation, Proc. Roy. Soc. Edinburgh Sect. A, 123 (1993), 433-460. 1

[2] D. Andreucci, G. R. Cirmi, S. Leonardi, A. F. Tedeev, Large time behavior of solutions to the Neumann problem for a quasilinear second order degenerate parabolic equation in domains with noncompact boundary, J. Differential Equations, 174 (2001), 253-288.1 
[3] K. Deng, H. A. Levine, The role of critical exponents in blow-up theorems: the sequel, J. Math. Anal. Appl., 243 (2000), 85-126. 1

[4] M. Fira, B. Kawohl, Large time behavior of solutions to a quasilinear parabolic equation with a nonlinear boundary condition, Adv. Math. Sci. Appl., 11 (2001), 113-126. 1

[5] A. Friedman, Partial differential equations of parabolic type, Prentice-Hall, Inc., Englewood Cliffs, N.J., (1964). 2

[6] H. Fujita, On the blowing up of solutions of the Cauchy problem for $\mathrm{u}_{\mathrm{t}}=\Delta \mathrm{u}+\mathrm{u}^{1+\alpha}$, J. Fac. Sci. Univ. Tokyo Sect. I, 13 (1966), 109-124. 1

[7] H. A. Levine, The role of critical exponents in blowup theorems, SIAM Rev., 32 (1990), 262-288. 1

[8] H. A. Levine, Q. S. Zhang, The critical Fujita number for a semilinear heat equation in exterior domains with homogeneous Neumann boundary values, Proc. Roy. Soc. Edinburgh Sect. A, 130 (2000), 591-602. 1

[9] P. Meier, On the critical exponent for reaction-diffusion equations, Arch. Rational Mech. Anal., 109 (1990), 63-71. 1

[10] Y.-W. Qi, The critical exponents of parabolic equations and blow-up in $\mathbf{R}^{\mathrm{n}}$, Proc. Roy. Soc. Edinburgh Sect. A, 128 (1998), 123-136. 1

[11] Y.-W. Qi, M.-X. Wang, Critical exponents of quasilinear parabolic equations, J. Math. Anal. Appl., 267 (2002), $264-280$. 1

[12] Z.-J. Wang, J.-X. Yin, C.-P. Wang, Critical exponents of the non-Newtonian polytropic filtration equation with nonlinear boundary condition, Appl. Math. Lett., 20 (2007), 142-147.

[13] C.-P. Wang, S.-N. Zheng, Critical Fujita exponents of degenerate and singular parabolic equations, Proc. Roy. Soc. Edinburgh Sect. A, 136 (2006), 415-430. 1

[14] C.-P. Wang, S.-N. Zheng, Fujita-type theorems for a class of nonlinear diffusion equations, Differential Integral Equations, 26 (2013), 555-570.

[15] C.-P. Wang, S.-N. Zheng, Z.-J. Wang, Critical Fujita exponents for a class of quasilinear equations with homogeneous Neumann boundary data, Nonlinearity, 20 (2007), 1343-1359.

[16] M. Winkler, A critical exponent in a degenerate parabolic equation, Math. Methods Appl. Sci., 25 (2002), 911-925.

[17] Q. S. Zhang, A general blow-up result on nonlinear boundary-value problems on exterior domains, Proc. Roy. Soc. Edinburgh Sect. A, 131 (2001), 451-475.

[18] S.-N. Zheng, X.-F. Song, Z.-X. Jiang, Critical Fujita exponents for degenerate parabolic equations coupled via nonlinear boundary flux, J. Math. Anal. Appl., 298 (2004), 308-324. 1

[19] S.-N. Zheng, C.-P. Wang, Large time behaviour of solutions to a class of quasilinear parabolic equations with convection terms, Nonlinearity, 21 (2008), 2179-2200. 1, 3, 3.4

[20] Q. Zhou, Y.-Y. Nie, X.-Y. Han, Large time behavior of solutions to semilinear parabolic equations with gradient, J. Dyn. Control Syst., 22 (2016), 191-205. 1, 3, 3.4, 3.4 